\title{
Inline Index Helped in Cleaning up Data Contamination Generated During Library Preparation and the Subsequent Steps
}

\author{
Ying Wang ( $\square$ 452985892@qq.com) \\ Shanghai Ocean University https://orcid.org/0000-0002-0183-7211 \\ Hao Yuan \\ Shanghai Ocean University \\ Junman Huang \\ Shanghai Ocean University \\ Chenhong Li \\ Shanghai Ocean University https://orcid.org/0000-0003-3075-1756
}

\section{Research Article}

Keywords: Contamination, cross-contamination, indexing, next-generation sequencing, environmental DNA, phylogenomics, meta genomics, multiplex

Posted Date: September 3rd, 2021

DOI: https://doi.org/10.21203/rs.3.rs-840856/v1

License: (1) This work is licensed under a Creative Commons Attribution 4.0 International License. Read Full License

Version of Record: A version of this preprint was published at Molecular Biology Reports on October 29th, 2021. See the published version at https://doi.org/10.1007/s11033-021-06884-y. 


\section{Abstract}

High-throughput sequencing involves library preparation and amplification steps, which may induce contamination across samples or between samples and the environment. We tested the effect of applying an inline-index strategy, in which DNA indices of 6 bp were added to both ends of the inserts at the ligation step of library prep for resolving the data contamination problem. Our results showed that the contamination ranged from $0.29-1.25 \%$ in one experiment and from $0.83-27.01 \%$ in the other. We also found that contamination could be environmental or from reagents besides cross-contamination between samples. Inline-index method is a useful experimental design to clean up the data and address the contamination problem which has been plaguing high-throughput sequencing data in many applications.

\section{Introduction}

High-throughput sequencing has become a dominant and efficient data collection method for researches, such as phylogenomics, environmental DNA and ancient DNA analyses. However, contamination from the environment or cross-contamination from other samples has become a headache in many studies involving high-throughput sequencing [1-5]. Contaminated data may deeply bias results of phylogenetic analysis [2, 6], over-estimate branch length and affect orthology detection [7]. Contamination also can confound metagenomic studies [1]. For example, distinct microbial community of placenta was proven to be an artifact due to contamination [8]. In ancient DNA analysis, pollution of modern human DNA can bias the estimates [9], whereas unexpected amplification of negative control samples was often reported in eDNA analyses, due to the complex nature the eDNA samples [10-12].

Contamination can originate from environmental sources, such as extraction kits, plastic consumables and reagents, or accidently transferred from other samples of the same batch $[1,13]$. Synthesizing barcodes or outsourcing sequencing can also result in cross-contamination which is beyond the control of the researcher's lab [14]. Many steps of washing and amplification are involved when preparing library for highthroughput sequencing which may increase the risk of contamination. If further manipulation of the samples is applied, such as target-gene enrichment, higher chance of contamination often occur.

Strict decontamination protocols and guidelines for preventing contamination in eDNA experiment have been proposed [15]. A standard checklist also was suggested for metagenomic lab for avoiding contamination [1]. Furthermore, various methods were designed for detecting contamination $[14,16]$ and different pipelines and tools were created to clean up the contaminated data $[10,13,14,16-19]$. Nevertheless, these methods are either using precautions to reduce the chance of contamination or identifying contaminator posteriorly though comparing the reads to references or other samples.

Rohland et al. (2015) [20] designed incomplete adapters composing P5/P7 barcodes (inline index) and partial Illumina adapters to increase efficacy of target enrichment. They also suggested that the P5/P7 barcodes can be used to trace cross-contamination, however, they did not test this idea. Here, we applied the "inline-index" strategy on two representative datasets, one from a phylogenomic study and the other for environment DNA data. Our objective is to test with empirical data on how the inline-index strategy can be used to solve the problem of cross contamination.

\section{Materials \& Methods}

\subsection{Designing and synthesizing the inline indices}

Indices of 6 bp were designed using scripts downloaded from the website (https://bioinf.eva.mpg.de/multiplex/, accessed on May 2018). The following filters were applied: 1) editing distance was 3, i.e., at least three substitutions should occur for one index changing to another, so to avoid index swap due to amplification or sequencing errors; 2) no polymer index was used, so to distinguish index signal from dust or chemical particles; 3) exclude the following sequences, 'AAA', 'ACA', 'CCC', 'CAC', 'GGG', 'GTG', 'TTT', and 'TGT' to avoid using the nucleotides that can be illuminated with the same laser consecutively. The inline indices were added to the 3 ' end of the IS1 or IS2 sequences of Meyer and Kircher (2010)[21]. Reverse and complementary oligos to the inline indices were added to the $5^{\prime}$ of their IS3 sequences (Fig. 1a). Twentyfour pairs of oligos (IS1_IndL and IS3_IndL') with inline indices were designed and synthesized for making P5 adapter and 24 pairs (IS2_IndR and IS3_IndR') were done for P7 adapter (table 1). The inline indices were synthesized at Sangon Biotech (Shanghai, China). Protocol of Meyer and Kircher (2010) [21] was followed to mix the oligos to make P5 and P7 adapters. It should be noticed that each IS1_IndL and IS2_IndR should be paired with its corresponding reverse and complementary IS3. Extreme precaution should be taken to avoid contamination during index preparation. The prepared indices should be aliquoted to small portions and stored under $-20^{\circ} \mathrm{C}$.

Table 1

The oligos for making adapters with inline indices. 


\begin{tabular}{|c|c|c|c|c|}
\hline TCTGCC & IS1_Ind1 & $\mathrm{A}^{\star} \mathrm{C}^{\star} \mathrm{A}^{\star} \mathrm{C}^{\star}$ TCTTTCCCTACACGACGCTCTTCCGATCTtc $\mathrm{C}^{\star}{ }^{\star} \mathrm{g}^{*} \mathrm{C}^{\star} \mathrm{C}$ & IS3_Ind1 & ggcagaAGATCGGAA*G*A*G*C \\
\hline GTCTCT & IS1_Ind2 & $\mathrm{A}^{\star} \mathrm{C}^{\star} \mathrm{A}^{\star} \mathrm{C}^{\star}{ }^{*}$ CTTTCCCTACACGACGCTCTTCCGATCTg ${ }^{*} \mathrm{C}^{\star} \mathrm{A}^{\star} \mathrm{C}^{\star} \mathrm{t}$ & IS3_Ind2 & agagacAGATCGGAA*G*A*G*C \\
\hline ATATTG & 1_Ind3 & $A^{*} C^{\star} A^{*} C^{\star}$ TCTTTCCCTACACGACGCTCTTCCGATCTat*a*t*t*g & IS3_Ind3 & caatatAGATCGGAA* $\mathrm{A}^{*} \mathrm{~A}^{*} \mathrm{G}{ }^{*} \mathrm{C}$ \\
\hline TGGAAG & S1_Ind4 & ${ }^{*} \mathrm{C}^{\star} \mathrm{A}^{\star} \mathrm{C}^{\star}$ TCTTTCCCTACACGACGCTCTTCCGATCTtg* $\mathrm{g}^{\star} \mathrm{a}^{\star} \mathrm{a}^{\star} \mathrm{g}$ & IS3_Ind4 & cttccaAGATCGGAA* $G^{\star} A^{*} G^{*} C$ \\
\hline TCTAGT & IS1_Ind5 & $\mathrm{A}^{\star} \mathrm{C}^{\star} \mathrm{A}^{\star} \mathrm{C}^{\star}$ TCTTTCCCTACACGACGCTCTTCCGATCTtc* ${ }^{\star \star} \mathrm{a}^{\star} \mathrm{g}^{\star} \mathrm{t}$ & IS3_Ind5 & actagaAGATCGGAA* ${ }^{*} A^{*} G^{*} C$ \\
\hline AGAGTA & IS1_Ind6 & $A^{\star} C^{\star} A^{\star} C^{\star}$ TCTTTCCCTACACGACGCTCTTCCGATCTag* $a^{\star} g^{\star \star}{ }^{\star} a$ & IS3_Ind6 & tactctAGATCGGAA* $\mathrm{G}^{*} \mathrm{~A}^{*} \mathrm{G}^{*} \mathrm{C}$ \\
\hline GGCCAA & IS1_Ind7 & $A^{*} C^{\star} A^{*} C^{\star}$ TCTTTCCCTACACGACGCTCTTCCGATCTgg ${ }^{*} C^{*} C^{*} a^{* a}$ & IS3_Ind7 & $\operatorname{ttggcc} A G A T C G G A A^{*} G^{*} A^{*} G^{\star} C$ \\
\hline TATCTC & IS1_Ind8 & 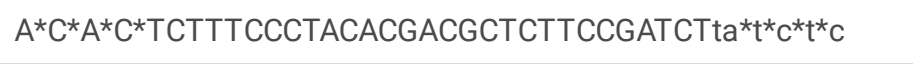 & IS3_Ind8 & gagataAGATCGGAA* ${ }^{*} A * G * C$ \\
\hline TTATGC & IS1_Ind9 & $\mathrm{A}^{\star} \mathrm{C}^{\star} \mathrm{A}^{\star} \mathrm{C}^{\star} \mathrm{TCTTTCCCTACACGACGCTCTTCCGATCTtt*}^{\star} \mathrm{a}^{\star t^{\star}} \mathrm{g}^{\star} \mathrm{C}$ & IS3_Ind9 & gcataaAGATCGGAA* ${ }^{*} A^{*} G^{*} C$ \\
\hline AGTTGG & IS1_Ind10 & $A^{*} C^{\star} A^{*} C^{\star}$ TCTTTCCCTACACGACGCTCTTCCGATCTag*t*t* $g^{\star} g$ & IS3_Ind10 & ccaactAGATCGGAA* $G^{*} A^{*} G^{*} C$ \\
\hline GTCAAG & IS1_Ind11 & $A^{*} C^{\star} A^{\star} C^{*}$ TCTTTCCCTACACGACGCTCTTCCGATCTgt* $C^{\star} a^{*} a^{\star} g$ & IS3_Ind11 & cttgacAGATCGGAA* $G^{\star} A^{*} G^{\star} C$ \\
\hline CAGCAA & IS1_Ind12 & $A^{*} C^{\star} A^{*} C^{\star}$ TCTTTCCCTACACGACGCTCTTCCGATCTCa* $g^{*} C^{\star} a^{\star} a$ & IS3_Ind12 & $\operatorname{ttg} \operatorname{ctg} A G A T C G G A A^{*} G^{*} A * G * C$ \\
\hline TCGCCG & IS1_Ind13 & $A^{*} C^{\star} A^{*} C^{*}$ TCTTTCCCTACACGACGCTCTTCCGATCTtC* $\mathrm{g}^{*} \mathrm{C}^{*} \mathrm{C}^{\star} \mathrm{g}$ & IS3_Ind13 & $\operatorname{cgg} c g a A G A T C G G A A^{*} G^{*} A^{*} G * C$ \\
\hline CTAAGA & IS1_Ind14 & $A^{*} C^{\star} A^{\star} C^{\star}$ TCTTTCCCTACACGACGCTCTTCCGATCTCt*a*a* $g^{\star} a$ & IS3_Ind14 & tcttagAGATCGGAA* $\mathrm{G}^{*} \mathrm{~A}^{*} \mathrm{G} * \mathrm{C}$ \\
\hline CCGCTT & IS1_Ind15 & $A^{*} C^{\star} A^{*} C^{\star}$ TCTTTCCCTACACGACGCTCTTCCGATCTCC ${ }^{*}{ }^{*} \mathrm{C}^{\star} \mathrm{t}^{\star} \mathrm{t}$ & IS3_Ind15 & aagcggAGATCGGAA*G* $A^{*} G^{*} C$ \\
\hline AAGTTA & IS1_Ind16 & $A^{*} C^{\star} A^{\star} C^{\star}$ TCTTTCCCTACACGACGCTCTTCCGATCTaa* $g^{\star} t^{\star} t^{\star} a$ & IS3_Ind16 & taacttAGATCGGAA*G*A*G*C \\
\hline GGTACC & IS1_Ind17 & $A^{*} C^{\star} A^{*} C^{*}$ TCTTTCCCTACACGACGCTCTTCCGATCTgg ${ }^{\star} t^{\star} a^{*} C^{\star} C$ & IS3_Ind17 & ggtaccAGATCGGAA* $G^{*} A^{\star} G * C$ \\
\hline CCAGGT & IS1_Ind18 & $A^{\star} C^{\star} A^{\star} C^{\star}$ TCTTTCCCTACACGACGCTCTTCCGATCTCC ${ }^{\star}{ }^{*} g^{\star} g^{\star} t$ & IS3_Ind18 & $\operatorname{acctgg} A G A T C G G A A^{*} G^{*} A^{*} G * C$ \\
\hline AATCGA & IS1_Ind19 & $A^{*} C^{\star} A^{*} C^{\star}$ TCTTTCCCTACACGACGCTCTTCCGATCTaa* ${ }^{*} \mathrm{C}^{\star} \mathrm{g}^{\star} \mathrm{a}$ & IS3_Ind19 & tcgattAGATCGGAA*G*A*G*C \\
\hline AACGCA & IS1_Ind20 & $A^{*} C^{*} A^{*} C^{*}$ TCTTTCCCTACACGACGCTCTTCCGATCTaa* ${ }^{*} \mathrm{~g}^{*} \mathrm{C}^{\star} \mathrm{a}$ & IS3_Ind20 & $\operatorname{tgcgttAGATCGGAA}{ }^{*} \mathrm{~A}^{\star} \mathrm{G}^{*} \mathrm{C}$ \\
\hline GACGAC & IS1_Ind21 & $A^{*} C^{\star} A^{*} C^{*}$ TCTTTCCCTACACGACGCTCTTCCGATCTga* ${ }^{*} g^{*} a^{*} C$ & IS3_Ind21 & gtcgtcAGATCGGAA* $G * A * G * C$ \\
\hline CGCGCT & IS1_Ind22 & $A^{\star} C^{\star} A^{\star} C^{\star}$ TCTTTCCCTACACGACGCTCTTCCGATCTCg ${ }^{*} C^{\star} g^{\star} C^{\star} t$ & IS3_Ind22 & $\operatorname{agcgcg} A G A T C G G A A^{\star} G^{*} A^{*} G^{*} C$ \\
\hline CCGTAG & IS1_Ind23 & $A^{*} C^{\star} A^{\star} C^{\star}$ TCTTTCCCTACACGACGCTCTTCCGATCTCC* $g^{\star} t^{\star} a^{\star} g$ & IS3_Ind23 & ctacggAGATCGGAA* $G^{*} A^{\star} G^{*} C$ \\
\hline \multirow[t]{2}{*}{ GTAATC } & IS1_Ind24 & $A^{*} C^{\star} A^{\star} C^{\star}$ TCTTTCCCTACACGACGCTCTTCCGATCTgt*a*a* & IS3_Ind24 & $A * G * A * G * C$ \\
\hline & IS2 IndR & & IS3 IndR' & \\
\hline GACCTT & IS2_Ind25 & $* T * G * \Delta * C T G C A G T T C A G A C G T G T G C T C T T C C G \Delta T C T g a *$ & IS3_Ind25 & aaggtcAGATCGGAA* $\mathrm{A}^{\star} A^{\star} \mathrm{G}^{\star} \mathrm{C}$ \\
\hline TCATAA & IS2_Ind26 & $\mathrm{G}^{\star} \mathrm{T}^{\star} \mathrm{G}^{\star} \mathrm{A}^{\star}$ CTGGAGTTCAGACGTGTGCTCTTCCGATCTtc*a*t*a*a & IS3_Ind26 & ttatgaAGATCGGAA*G*A*G*C \\
\hline CAAGAG & IS2_Ind27 & $\mathrm{G}^{*} \mathrm{~T}{ }^{*} \mathrm{G} \mathrm{A}^{*}$ CTGGAGTTCAGACGTGTGCTCTTCCGATCTCa*a* $\mathrm{g}{ }^{*}{ }^{*} \mathrm{~g}$ & IS3_Ind27 & $\operatorname{ctcttg} A G A T C G G A A^{*} G^{*} A^{*} G^{*} C$ \\
\hline CGATCA & IS2_Ind28 & ${ }^{*} T^{\star} G^{*} A^{*}$ CTGGAGTTCAGACGTGTGCTCTTCCGATCTCg*a*t* ${ }^{\star}{ }^{\star} a$ & IS3_Ind28 & $\operatorname{tgatcg} A G A T C G G A A^{*} G^{\star} A^{*} G^{*} C$ \\
\hline TTGATT & IS2_Ind29 & $\mathrm{G}^{*} T^{\star} \mathrm{G}^{*} \mathrm{~A}^{\star}$ CTGGAGTTCAGACGTGTGCTCTTCCGATCTtt*g*a*t*t & IS3_Ind29 & aatcaaAGATCGGAA* ${ }^{\star} A^{*} G^{*} C$ \\
\hline TCCGAG & IS2_Ind30 & $\mathrm{G}^{*} \mathrm{~T}^{*} \mathrm{G}^{*} \mathrm{~A}^{*}$ CTGGAGTTCAGACGTGTGCTCTTCCGATCTIC* $\mathrm{C}^{*} \mathrm{~g}^{*} \mathrm{a}^{\star} \mathrm{g}$ & IS3_Ind30 & $\operatorname{ctcggaAGATCGGA} A^{*} G^{*} A^{*} G^{*} C$ \\
\hline CCTGAA & IS2_Ind31 & $\mathrm{G}^{*} \mathrm{~T}^{\star} \mathrm{G}^{\star} \mathrm{A}^{\star}$ CTGGAGTTCAGACGTGTGCTCTTCCGATCTC ${ }^{\star} \mathrm{t}^{\star} \mathrm{g}^{\star} \mathrm{a}^{\star} \mathrm{a}$ & IS3_Ind31 & ttcaggAGATCGGAA* $G^{\star} A^{*} G^{*} C$ \\
\hline ATTCTT & IS2_Ind32 & 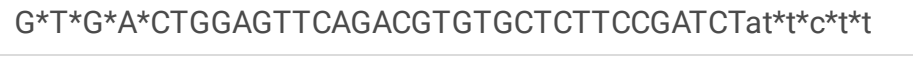 & IS3_Ind32 & aagaatAGATCGGAA* $\mathrm{G}^{\star} A^{\star} \mathrm{G}^{*} \mathrm{C}$ \\
\hline GCGACT & IS2_Ind33 & $\mathrm{G}^{\star} T^{\star} \mathrm{G}^{\star} \mathrm{A}^{\star} \mathrm{CTGGAGTTCAGACGTGTGCTCTTCCGATCTg}{ }^{\star} \mathrm{g}^{\star} \mathrm{a}^{\star} \mathrm{C}^{\star} \mathrm{t}$ & IS3_Ind33 & $\operatorname{agtcgcAGATCGGA} A^{*} G^{\star} A^{*} G^{*} C$ \\
\hline GGCTTC & IS2_Ind34 & $\mathrm{G}^{*} T^{\star} \mathrm{G}^{\star} \mathrm{A}^{\star} \mathrm{CTGGAGTTCAGACGTGTGCTCTTCCGATCTgg}{ }^{*} \mathrm{C}^{\star} \mathrm{t}^{\star} \mathrm{t}^{\star} \mathrm{C}$ & IS3_Ind34 & gaagccAGATCGGAA* $G^{*} A^{*} G^{*} C$ \\
\hline AATACG & IS2_Ind35 & 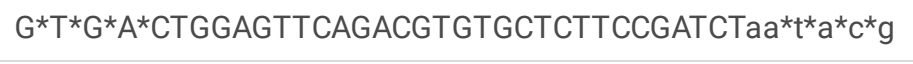 & IS3_Ind35 & cgtattAGATCGGAA* $G^{*} A^{*} G^{*} C$ \\
\hline TACGGT & IS2_Ind36 & $\mathrm{G}^{\star} \mathrm{T}^{*} \mathrm{G}^{\star} \mathrm{A}^{*} \mathrm{CTGGAGTTCAGACGTGTGCTCTTCCGATCTta}{ }^{*} \mathrm{C}^{\star} \mathrm{g}^{*} \mathrm{~g}^{\star \mathrm{t}}$ & IS3_Ind36 & $\operatorname{accgtaAGATCGGAA}{ }^{*}{ }^{*} A^{\star} G * C$ \\
\hline
\end{tabular}




\begin{tabular}{|c|c|c|c|c|}
\hline ACCGTC & IS2_Ind37 & $\mathrm{G} * T * G * A * C T G G A G T T C A G A C G T G T G C T C T T C C G A T C T a{ }^{*}{ }^{*} g^{*}{ }^{*} \mathrm{C}$ & IS3_Ind37 & gacggtAGATCGGAA*G*A*G*C \\
\hline AGAAGC & IS2_Ind38 & $\mathrm{G} * T * G * A * C T G G A G T T C A G A C G T G T G C T C T T C C G A T C T a g * a * a * g * C$ & IS3_Ind38 & gcttctAGATCGGAA*G*A*G*C \\
\hline CATAGC & IS2_Ind39 & $\mathrm{G} * T * G * A * C T G G A G T T C A G A C G T G T G C T C T T C C G A T C T c a * t^{\star} a * g{ }^{*} \mathrm{C}$ & IS3_Ind39 & gctatgAGATCGGAA* ${ }^{*} A^{*} G{ }^{*} C$ \\
\hline AGGCTC & IS2_Ind40 & $\mathrm{G} * T^{\star} \mathrm{G}^{*} \mathrm{~A}^{*} \mathrm{CTGGAGTTCAGACGTGTGCTCTTCCGATCTag}{ }^{*}{ }^{*} \mathrm{C}^{\star} \mathrm{t}^{\star} \mathrm{C}$ & IS3_Ind40 & gagcctAGATCGGAA* ${ }^{*} A * G * C$ \\
\hline CTGCGG & IS2_Ind41 & $\mathrm{G} * T * G * A * C T G G A G T T C A G A C G T G T G C T C T T C C G A T C T c{ }^{*} g^{\star} C^{*} g * g$ & IS3_Ind41 & ccgcagAGATCGGAA*G*A*G*C \\
\hline CTCGGC & IS2_Ind42 & 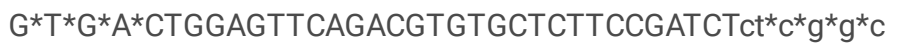 & IS3_Ind42 & gccgagAGATCGGAA*G*A*G*C \\
\hline GATTAG & IS2_Ind43 & $\mathrm{G} * T * G * A * C T G G A G T T C A G A C G T G T G C T C T T C C G A T C T g a * t * t^{\star} a * g$ & IS3_Ind43 & ctaatcAGATCGGAA*G*A*G*C \\
\hline AGATAT & IS2_Ind44 & $\mathrm{G} * T^{\star} \mathrm{G}^{\star} \mathrm{A}{ }^{\star}$ CTGGAGTTCAGACGTGTGCTCTTCCGATCTag*a*t*a*t & IS3_Ind44 & atatctAGATCGGAA* ${ }^{\star} A^{*} \mathrm{G} * \mathrm{C}$ \\
\hline TGGTCC & IS2_Ind45 & $\mathrm{G} * T * G{ }^{*} \mathrm{~A} * \mathrm{CTGGAGTTCAGACGTGTGCTCTTCCGATCTtg}{ }^{*}{ }^{\star}{ }^{*} \mathrm{C}^{*} \mathrm{C}$ & IS3_Ind45 & ggaccaAGATCGGAA*G* $A * G * C$ \\
\hline GTTCCG & IS2_Ind46 & $\mathrm{G} * T * G * A * C T G G A G T T C A G A C G T G T G C T C T T C C G A T C T g t^{\star} t^{*} C^{*} C^{*} g$ & IS3_Ind46 & cggaacAGATCGGAA*G*A*G*C \\
\hline GTACGT & IS2_Ind47 & $\mathrm{G} * T * G * A * C T G G A G T T C A G A C G T G T G C T C T T C C G A T C T g{ }^{*} a * C * g * t$ & IS3_Ind47 & $\operatorname{acgtac} A G A T C G G A A * G * A * G * C$ \\
\hline AAGAAC & IS2_Ind48 & $\mathrm{G} * T * \mathrm{G} * A * \mathrm{CTGGAGTTCAGACGTGTGCTCTTCCGATCTaa*} \mathrm{g}{ }^{*} a * a{ }^{*}$ & IS3_Ind48 & gttcttAGATCGGAA*G*A*G*C \\
\hline
\end{tabular}

\subsection{Library preparation and sequencing}

Two batches of sequencing libraries were prepared. One was for a phylogenomic study of eight chondrichthyan species, Himantura uarnak, Chiloscyllium griseum, Rhinobatos annandalei, Tetronarce nobiliana, Rhina ancylostoma, Neoharriotta pinnata, Pristiophorus cirratus, Heterodontus francisci. The genomic DNA was extracted from tissue samples using Ezup DNA extraction kits (B518251, Sangon Biotech, Shanghai, China). The other batch of samples was 11 eDNA samples collected from the Red River, Yunnan, China. The water samples were filtered with $3.0 \mu \mathrm{m}$ PCTE membrane on site and DNA was extracted following Lin et al. (2019) [22]. The extracted DNA samples were sheared with a Covaries M220 focused-ultrasonicator to 250 bp (Covaris Inc., Woburn, Massachusetts). The protocol of Meyer and Kircher (2010) [21] was followed to prepare the sequencing libraries. For each sample, a unique pair of P5 and P7 adapters with inline index was added, while the P7 adapters also have their own classic P7 index (Fig.1b). Two different persons prepared the two batches of samples to represent handling variation in the lab. The libraries were sequenced on an Illumina HiSeqX 10 platform by Genewiz (Genewiz, Inc, Shanghai, China). We chose these two batches of samples to represent the common high-throughput sequencing applications where contamination has being problematic.

\subsection{Data analyses}

The raw reads were trimmed with trim_galore v0.6.4 (https://www.bioinformatics.babraham.ac.uk/projects/trim_galore/) to remove the adapter sequences and low quality reads. The duplicated reads due to PCR amplification were excluded using the "-fastx_uniques" command in USEARCH v10.0.240 [23]. A custom Perl script (demultiplex_inline.pl, supplementary files) was used to extract the first 6 bp of the sequences, compare to the sequences of the inline index pairs used for samples.

The reads with both end matching the inline index pairs were consider as "true reads" and the rest reads were consider as "others". The "others" were further counted and separated into four part: 1) "cross-contamination" (contamination_cnt.pl, supplementary files), DNA fragments have inline index but instead of the correct one, they are used in other samples in the same batch; 2) "inline index mutation" (kmer_summary_1sub.py, supplementary files), the inline index sequences have one mutation to the correct one; 3 ) "inline index contamination" (kmer_summary.py, supplementary files), the sequences matched inline index used in our lab but not for the same batch of samples; 4) "unknown", all the rest reads cannot trace back to a source.

\section{Results}

\subsection{Composition of the reads}

The "true reads" that have correct inline index at both ends accounted for $38.16 \%$ to $80.04 \%$ of the total reads of each sample in the eDNA experiment (Fig. 2a). Sample 2 and 3 had substantial amount of contamination, probably due to operational errors dealing with these two samples. True reads consisted of $87.81 \%$ to $92.36 \%$ of total reads of each sample in the chondrichthyan study (Fig. $3 a$ ). 


\subsection{Correction of reads with index mutation}

Because reads with inline index mutation accounted for most part of the "other" reads, we corrected the index sequence to their closet indices used in our lab. After correction, the true reads increased to $48.67 \%$ to $90.51 \%$ for the eDNA experiment (Fig. 2 b) and $91.27 \%$ to $95.63 \%$ for the chondrichthyan experiment (Fig. 3b).

\subsection{Cross-contamination}

Contamination from other samples accounted for $0.83 \%-27.01 \%$ in the eDNA experiment and $0.29 \%-1.25 \%$ in the phylogenomic study of chondrichthyan (Fig. 4). The results showed that cross-contamination ubiquitously exists no matter who run the experiment and whether the tubes were close to each other or not on the rack, so the contaminator did not necessarily jump from adjacent tubes (Fig. 5).

\subsection{Inline index contamination}

Some reads had at least one inline index that was not used in the same batch of samples, but can be found in other inline indices use in our lab. Those could be due to cross-contamination between indices when they were synthesized. The eDNA samples had $1.21 \%$ to $12.1 \%$ contamination due to inline index mixing, whereas the chondrichthyan samples had $1.16 \%$ to $2.71 \%$ (Fig. 4).

\subsection{Unknown contamination}

Besides the cross-contamination among samples and inline index contamination, there were a large portion of reads with unknown sources, which accounted for $6.9 \%$ to $20.3 \%$ in the eDNA samples and $4.07 \%$ to $7.47 \%$ in the chondrichthyan samples (Fig. 3 and 4 ). The unknown contamination was randomly distributed among samples.

\section{Discussion}

The results of "true reads" and "others" showed that the baseline contamination in our lab is around $10 \%$ to $20 \%$, but an accidental handling can increase the number to almost $40 \%$. Allio et al.(2020)[7] found very low cross-contamination (0.26\%) in general in their genome shotgun sequencing data of swallowtail butterfly, but one sample, Parnassius imperator had a significant higher value, $26.71 \%$ of the contigs were contaminated, suggesting that human errors can have sporadic but severe impact on high-throughput sequencing data.

We found that the "others" had similar composition between the two experiments. If we disregard the sample 2 and 3 of the eDNA experiment, the composition of the "others", ranked from high to low was "inline index mutation", the "unknown", "index contamination" and "crosscontamination".

Because we applied a substitution distance of three base pairs, it should be safe to assign the reads with one base-pair mutation in their inline indices back to the "correct" sample. Indeed, we found few inline index had two substitutions. The index mutation could be caused by two reasons. One is sequencing error, which should be minimal because of the low error rate in sequencing the first six base pairs. The other error could happen while synthesizing the inline indices, so we should test the accuracy of synthesized indices from different provider in future.

The results of contaminated reads showed that the source of contamination was from sample 10, 7 and 1 in the eDNA experiment and from sample 8,7 and 1 in the chondrichthyan study. The degree of cross-contamination also was not uniform. For example, sample 2 and 3 of the eDNA experiment had $24.93 \%$ and $27.01 \%$ contamination mainly from sample 10 , suggesting handling errors may be involved. The route of cross-contamination may be through tube lids, gloves, pipette tips or aerosol.

The eDNA sample 7 had $12.1 \%$ inline index contamination, and mostly from one pair of inline indices, suggesting those may be contaminated by other library, prepared in our lab. We suggest that the index should be synthesized once at a time, so inline index contamination could be reduced. Extreme care should be taken when making the adapters in the lab, and the adapters should be divided into small aliquot for storage.

There were at least one unknown "inline index" sequenced for those reads. Our lab also has been using classic protocol of library prep [21], which has no inline index applied. We suspect those libraries that made with the classic protocol may have polluted common reagents, which in turn contaminated the samples of this project. Therefore, the first 6 bp base pairs of the reads cannot match any inline indices. 
In the "others", reads with one mutation in the index can be corrected into its original sample, but reads with mixed inline index or unknown index sequence cannot be assigned to any sample and should be excluded from further analyses.

Most analytical approaches are based on data filtering. For example, Croco (Simion, 2018) [24] is a database independent method that can be used to trace cross-contamination from divergent specie, but difference between closely related organisms cannot be recognized.

ConFindr (Low, 2019) [17] identifies contaminated samples, if it contains more than one allele of core single-copy ribosomal protein genes. Dickins et al. (2014) [14] proposed a two-part pipeline to identify the contaminated samples based on unexpected number of variants and a phylogenetics approach.

Inline-index method, however, is independent on the sequence of samples, so there is no requirement on the similarity between samples to deduce the composition of the reads. The reads can be recognized using inline index even the sample contain DNA from unknown species, such as the eDNA data, but the analytical methods may not work in this situation. Kircher et al. (2012) [25] designed a double-index technique to detect jumping PCR by adding indices to both P5 and P7 of Illumina adapters. Peyrégne et al. (2020) [26] implied that the double-index method can be used to monitor contamination. However, the double-index are added before sequencing, there are a lot of chance that contamination can happened before that. Rohland et al. (2015) [20] invented the inline-index method that adding a pair of unique barcode to both end of the DNA insert to trace the contamination. Because they aimed at ancient DNA research, and paid most of their attention to the effects of unique barcode to damage rate, but did not test whether the inline-index approach can be used to mitigate problems of crosscontamination. Our research results showed that contamination occurred ubiquitously and the unique inline barcode can be used to trace the source of read contamination.

Furthermore, because samples are labeled with inline-index the before sending them to sequencing facility, cross-contamination resulted in sequencing center can be controlled. Cross-contaminated reads from other samples can be assigned back to their origin based on their inlineindex pair to rescue the data. Finally, with 24 pairs of inline indices, plus the P7 index, more samples can be multiplexed and sequenced in the same sequencing lane to save the cost.

\section{Conclusion}

The inline index method is experimental, so it could be a good complementary to the analytical approach, such as CroCo and ConFindr. The wildly use of the combination of these methods should be a routine in near future. Environment DNA samples have a nature of mixed source of DNA with various concentration, so it is impossible to know if the reads are genuine or from other samples by using the computational approach. In this scenario, inline index method or other experimental method probably is the only way to address the contamination problem. In future, we can test whether the "blunt end" and "ligation" step of library prep can be combined together without washing steps in between, so to add the inline index at the very first step of library prep to further eliminate the chance of cross-contamination.

\section{Declarations}

\section{Funding}

This work was supported by "Science and Technology Commission of Shanghai Municipality (19050501900)" and "Shanghai Academy of Environmental Sciences".

\section{Conflicts of interest/Competing interests}

CL, YW and HY are authors on a patent applied for by Shanghai Ocean University that covers the inline index technology (201811406204.X).

\section{Availability of data and material}

The raw reads have been uploaded in the SRA under Bioproject number PRJNA749868, PRJNA750567

\section{Code availability (software application or custom code)}

The custom codes can be found in online version of this article.

\section{Authors' contributions}


YW and $\mathrm{CL}$ conceived the research plan. YW and JH did the experiments and collected data. YW, HY and JH analyzed the data. YW and CL wrote the draft. All authors edited and approved the final version of the manuscript.

\section{Ethics approval}

All experimental procedures involving fish were approved by the Animal Ethics Committee of Shanghai Ocean University, China.

\section{Consent to participate (include appropriate statements)}

All authors consent to participate.

\section{Consent for publication (include appropriate statements)}

All authors consent for publication.

\section{Acknowledgements:}

We are grateful to Ms. Lifang Peng for helping with collecting water samples.

\section{References}

1. Eisenhofer R et al (2019) Contamination in Low Microbial Biomass Microbiome Studies: Issues and Recommendations. Trends Microbiol 27(2):105-117

2. Laurin-Lemay S, Brinkmann H, Philippe H (2012) Origin of land plants revisited in the light of sequence contamination and missing data. Curr Biol 22(15):R593-R594

3. Sepulveda AJ et al., The Elephant in the Lab (and Field): Contamination in Aquatic Environmental DNA Studies. Frontiers in Ecology and Evolution, 2020. 8(440)

4. Ballenghien M, Faivre N, Galtier N (2017) Patterns of cross-contamination in a multispecies population genomic project: detection, quantification, impact, and solutions. BMC Biol 15(1):25

5. Koutsovoulos, G., et al., No evidence for extensive horizontal gene transfer in the genome of the tardigrade < em > Hypsibius dujardini</em>. Proceedings of the National Academy of Sciences, 2016. 113(18): p. 5053-5058.

6. Philippe $\mathrm{H}$ et al (2011) Resolving difficult phylogenetic questions: why more sequences are not enough. PLoS Biol 9(3):e1000602

7. Allio R et al (2020) Whole Genome Shotgun Phylogenomics Resolves the Pattern and Timing of Swallowtail Butterfly Evolution. Syst Biol 69(1):38-60

8. Perez-Muñoz ME et al (2017) A critical assessment of the "sterile womb" and "in utero colonization" hypotheses: implications for research on the pioneer infant microbiome. Microbiome 5(1):48

9. Racimo F, Renaud G, Slatkin M (2016) Joint Estimation of Contamination, Error and Demography for Nuclear DNA from Ancient Humans. PLoS Genet 12(4):e1005972

10. Hänfling B et al (2016) Environmental DNA metabarcoding of lake fish communities reflects long-term data from established survey methods. Mol Ecol 25(13):3101-3119

11. Sepulveda AJ et al (2019) Adding invasive species biosurveillance to the U.S. Geological Survey streamgage network. Ecosphere 10(8):e02843

12. Serrao NR, Reid SM, Wilson CC (2018) Establishing detection thresholds for environmental DNA using receiver operator characteristic (ROC) curves. Conservation Genetics Resources 10(3):555-562

13. Glassing A et al (2016) Inherent bacterial DNA contamination of extraction and sequencing reagents may affect interpretation of microbiota in low bacterial biomass samples. Gut Pathogens 8(1):24

14. Dickins $B$ et al (2014) Controlling for contamination in re-sequencing studies with a reproducible web-based phylogenetic approach. Biotechniques 56(3):134-141

15. Goldberg CS et al (2016) Critical considerations for the application of environmental DNA methods to detect aquatic species. Methods Ecol Evol 7(11):1299-1307 
16. De Simone G et al (2020) Contaminations in (meta)genome data: An open issue for the scientific community. IUBMB Life 72(4):698-705

17. Low AJ et al (2019) ConFindr: rapid detection of intraspecies and cross-species contamination in bacterial whole-genome sequence data. PeerJ 7:e6995

18. Straube $\mathrm{N}$ et al (2018) A phylogenomic approach to reconstruct interrelationships of main clupeocephalan lineages with a critical discussion of morphological apomorphies. BMC Evol Biol 18(1):158

19. Flickinger M et al (2015) Correcting for Sample Contamination in Genotype Calling of DNA Sequence Data. Am J Hum Genet 97(2):284290

20. Rohland N et al (2015) Partial uracil-DNA-glycosylase treatment for screening of ancient DNA. Philos Trans R Soc Lond B Biol Sci 370(1660):20130624

21. Meyer M, Kircher M, Illumina sequencing library preparation for highly multiplexed target capture and sequencing. Cold Spring Harb Protoc (2010) 2010(6): p. pdb prot5448

22. Lin M, Zhang S, Yao M (2019) Effective detection of environmental DNA from the invasive American bullfrog. Biol Invasions 21(7):22552268

23. Edgar RC (2010) Search and clustering orders of magnitude faster than BLAST. Bioinformatics 26(19):2460-2461

24. Simion P et al (2018) A software tool 'CroCo' detects pervasive cross-species contamination in next generation sequencing data. BMC Biol 16(1):28

25. Kircher M, Sawyer S, Meyer M (2012) Double indexing overcomes inaccuracies in multiplex sequencing on the Illumina platform. Nucleic Acids Res 40(1):e3

26. Peyrégne S, Prüfer K (2020) Present-Day DNA Contamination in Ancient DNA Datasets. Bioessays 42(9):e2000081

\section{Figures}

(A)

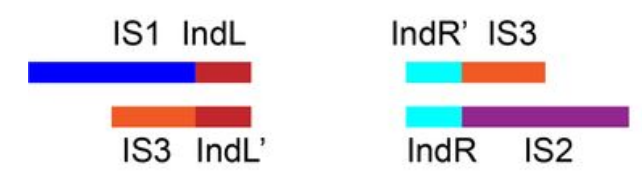

(B)

\section{DNA Sequence}

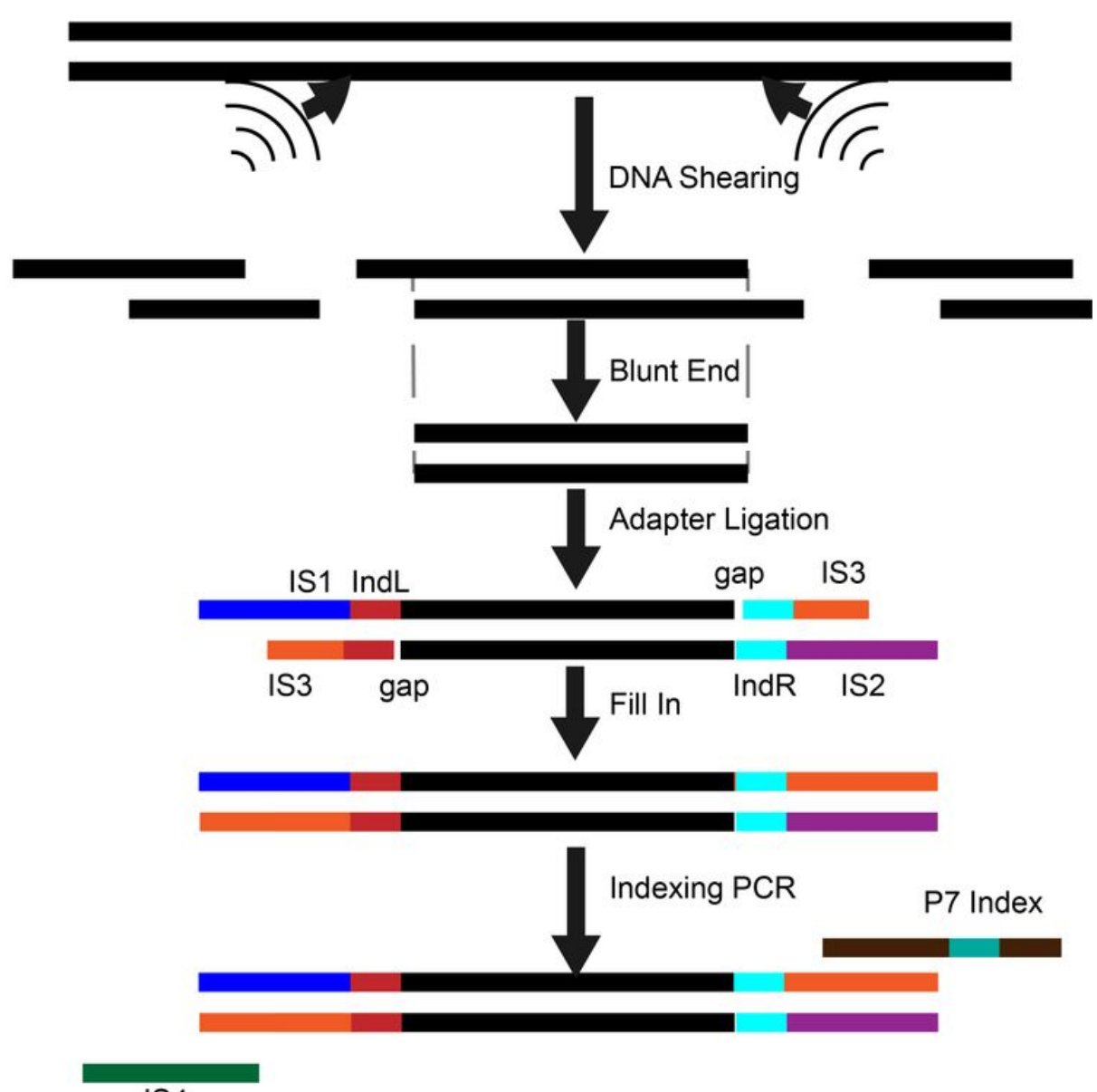

IS4 
Figure 1

Scheme of inline index and library preparation.

(A)

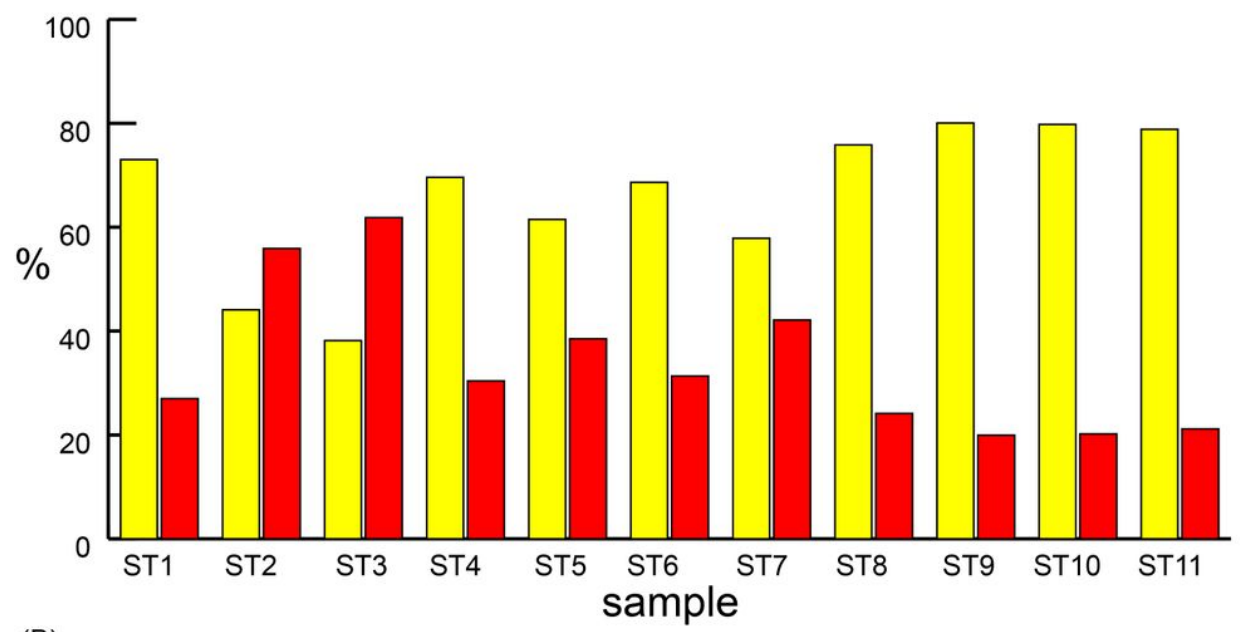

(B)

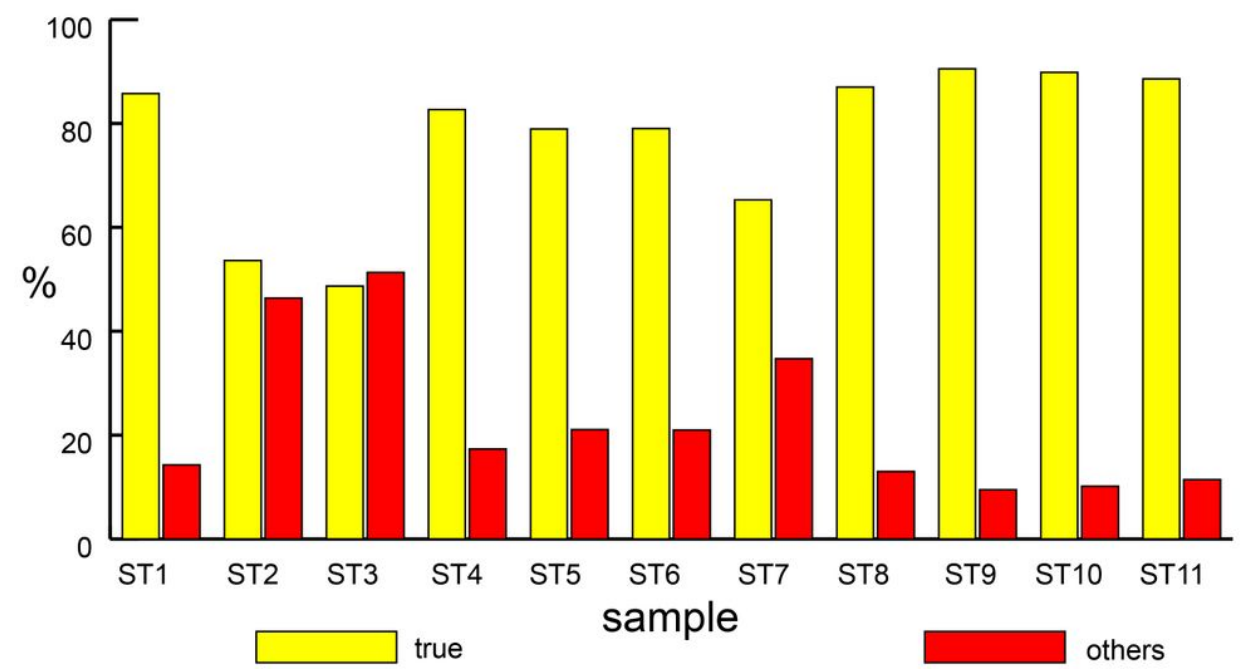

Figure 2

Percentage of reads assigned correctly in the eDNA experiment. A. before correction on reads with inline index mutation; B after correction on reads with inline index mutation. 


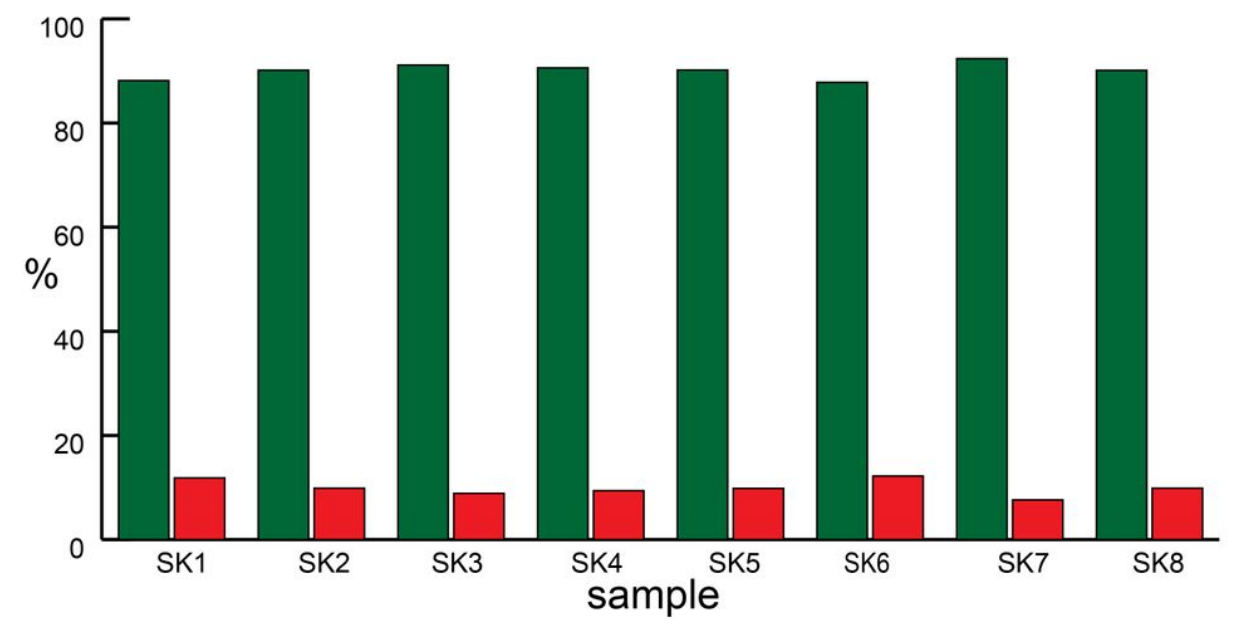

(B)

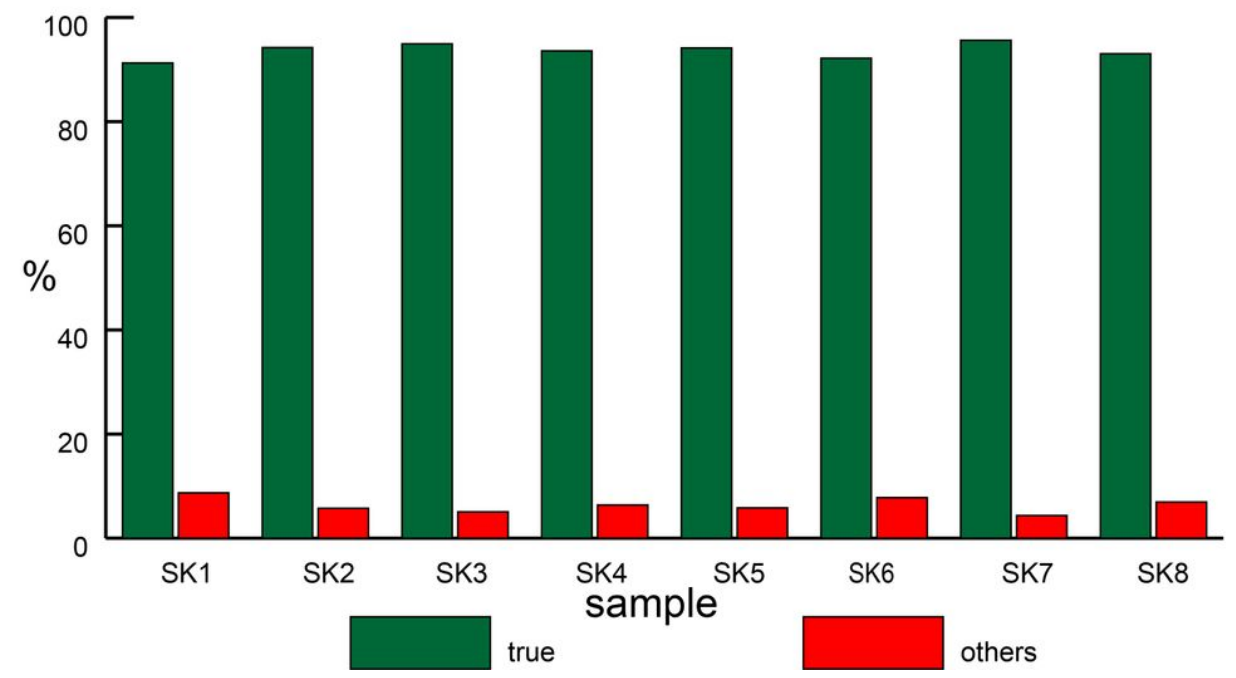

Figure 3

Percentage of reads assigned correctly in the chondrichthyan experiment. A. before correction on reads with inline index mutation; B after correction on reads with inline index mutation. 
(A)
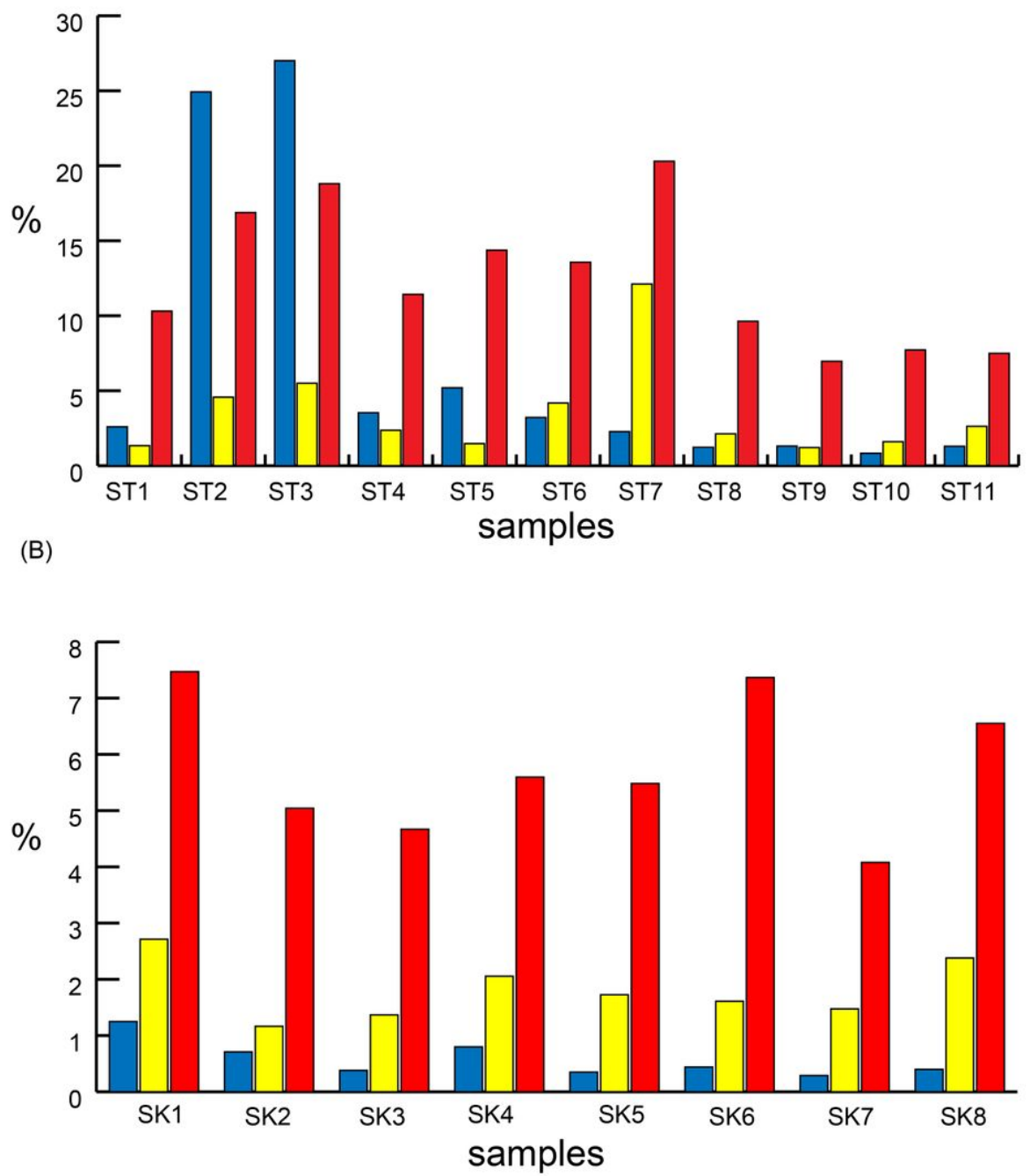

unknown contamination

index contamination

cross-contamination

Figure 4

Composition of the others reads after correction of inline index with one substitution. A. data from the eDNA experiment; B. data from the chondrichthyan experiment. 
(A)

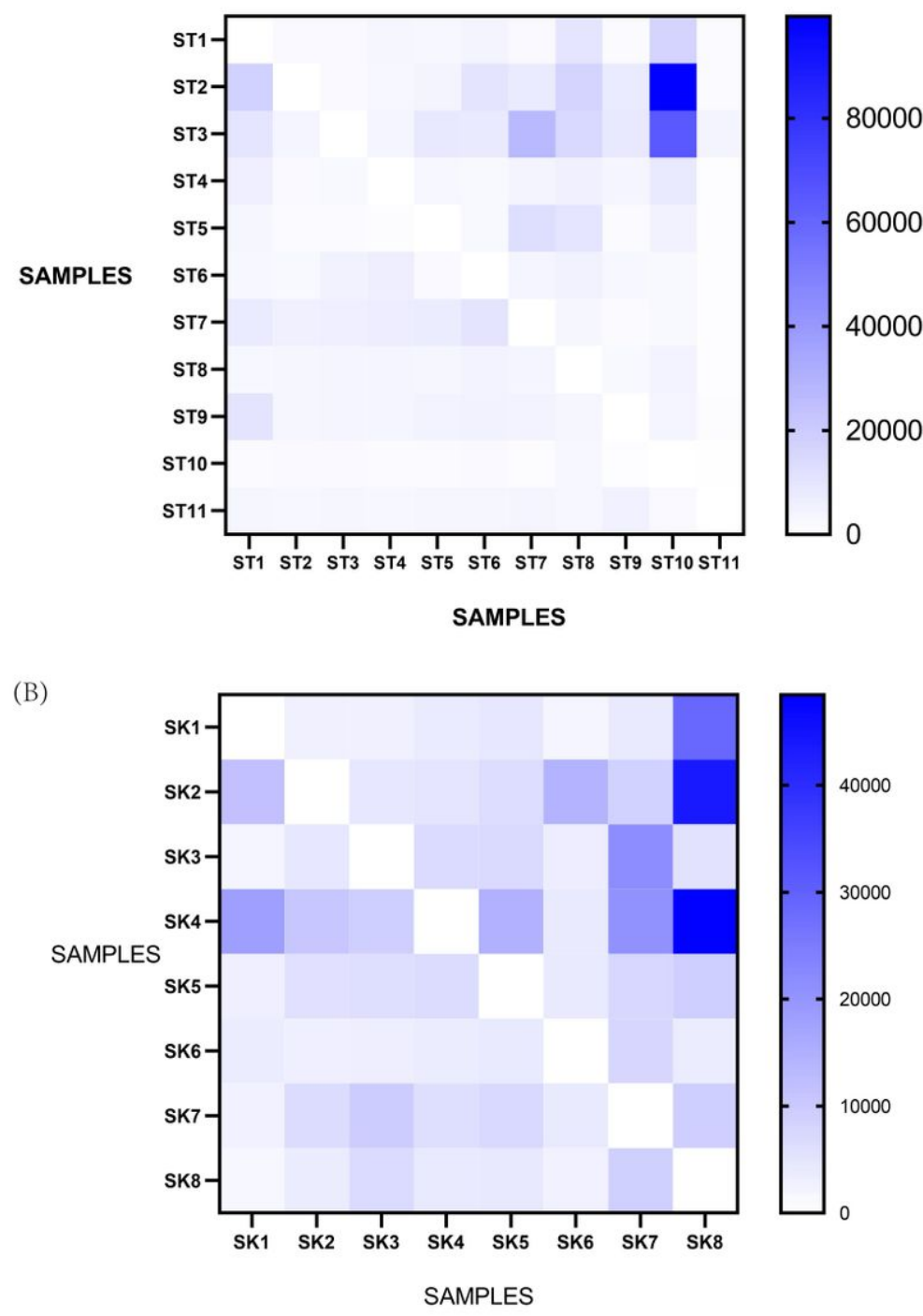

Figure 5

Cross-contamination among samples. A. in the eDNA experiment. B. in the chondrichthyan experiment.

\section{Supplementary Files}

This is a list of supplementary files associated with this preprint. Click to download.

- SupplementaryMaterialnoauthorinformation.doc 